\title{
Power-Handling Capabilities of Circular Dielectric Waveguide at Millimeter Wavelengths
}

\author{
DANIEL G. JABLONSKI, SENIOR MEMBER, IEEE
}

\begin{abstract}
The power-handling capabilities of a circular dielectric waveguide propagating the single $\mathrm{HE}_{11}$ mode are discussed. Simple calculations illustrate the limits imposed by dielectric heating and dielectric breakdown. Examples are presented for polystyrene and polytetrafluoroethylene (PTFE) guides at $70 \mathrm{GHz}$. The results suggest that maximum power levels for circular dielectric waveguide in the millimeter-wave spectrum will be of the orders of 10 to $100 \mathrm{~W}$ and that dielectric heating is the limiting phenomenon.
\end{abstract}

\section{INTRODUCTION}

$\mathrm{T}$ HERE HAS BEEN considerable study of the electromagnetic properties of a dielectric waveguide. However, there has been little, if any, mention of the powerhandling capabilities of such a guide. This is not surprising, as dielectric waveguide is primarily of interest at millimeter wavelengths, where levels of available power are rather low. Given the current amount of activity aimed at developing high-power millimeter wavelength sources, though, it seems useful to perform at least a simple study of the power limitations of a typical dielectric waveguide.

There are three physical phenomena that are of interest in this regard. The first, dielectric breakdown, is well known in conjunction with the use of dielectrics as highvoltage insulators. The second is dielectric heating, which results from power attenuation in the dielectric and can, in principle, cause the waveguide to melt. Finally, stimulated Raman and Brillouin scattering are of great concern to designers of optical-fiber systems. This is because the wavelength of a guided mode is sufficiently small for the optical signal to interact with lattice vibrations, defects, and impurities in the dielectric. However, scattering is of little importance at millimeter wavelengths, and is mentioned only because of the extremely close connection between the circular dielectric waveguide and an optical fiber.

Dielectric breakdown and dielectric heating are disussed in turn. Examples of dielectric breakdown and dielectric heating are given for various materials utilized as circular dielectric waveguide at $70 \mathrm{GHz}$. The discussion begins, however, with a brief review of the properties of the $\mathrm{HE}_{11}$ mode in a dielectric cylinder.

\footnotetext{
Manuscript received April 16, 1984; revised August 16, 1984. This work was supported by the Naval Surface Weapons Center under an Independent Research Grant.

The author is with the Naval Surface Weapons Center, White Oak, Silver Spring, MD 20910.
}

\section{Properties OF The Dielectric-RoD WAVEGUIDE}

The $\mathrm{HE}_{11}$ mode has received much attention, and only a few important points will be discussed here. A section of dielectric guide is sketched in Fig. 1. The guide consists of a cylinder of dielectric constant $\epsilon_{2}$ imbedded in a medium of dielectric constant $\epsilon_{1}$. Typically, the guide consists of a cylinder of polytetrafluoroethylene (PTFE) or polystyrene surrounded by air. Hence, $\epsilon_{1}$ will usually be equal to $\epsilon_{0}$, the free-space permittivity.

The field components of the allowed propagation modes are given by Stratton [1]. Provided that the radius $a$ of the cylinder is less than a critical radius $a_{0}$, only a single mode can propagate. This critical radius is shown by Cullen [2] to occur when

$$
J_{0}(x)=0
$$

where

$$
x=2 \pi a_{0}\left(\epsilon_{1} / \epsilon_{0}\right)^{1 / 2}\left(\epsilon_{r}-1\right)^{1 / 2} / \lambda_{0} .
$$

$J_{0}$ is the ordinary Bessel function of the first kind. The relative dielectric constant $\epsilon_{r}$ is defined as $\epsilon_{2} / \epsilon_{1}$, and $\lambda_{0}$ is the free-space wavelength of the guided wave.

As an example, a PTFE rod in air will have $\epsilon_{r} \simeq 2.08$. At $70 \mathrm{GHz}, \lambda_{0}=4.3 \mathrm{~mm}$. The first zero of $J_{0}$ occurs at $x=2.405$. Hence, the critical radius $a_{0}$ is found to be 1.58 $\mathrm{mm}$.

The single mode which propagates when $a<a_{0}$ is hybrid in nature and is known as the $\mathrm{HE}_{11}$ mode. There are longitudinal components of both $\mathrm{H}$ and $\mathrm{E}$ for this mode.

In principle, the $\mathrm{HE}_{11}$ mode can propagate regardless of how small the radius of the guide is. However, as the rod size is decreased, more and more of the energy in the guided wave propagates in the evanescent fields outside the guide. This is important in connection with the attenuation characteristics of the guide. In most instances, the dielectric rod has a non-negligible loss tangent $\tan \delta$, whereas the external medium is usually assumed to be loss free. Consequently, the net power attenuation will depend both on tan $\delta$ and on the relative amounts of power propagating inside and outside of the rod.

As an example, a wave propagating in an infinite dielectric $\epsilon_{r}$ has a power attenuation factor given by

$$
\alpha=\frac{2 \pi}{\lambda_{0}}\left(\epsilon_{r}\right)^{1 / 2} \tan \delta .
$$




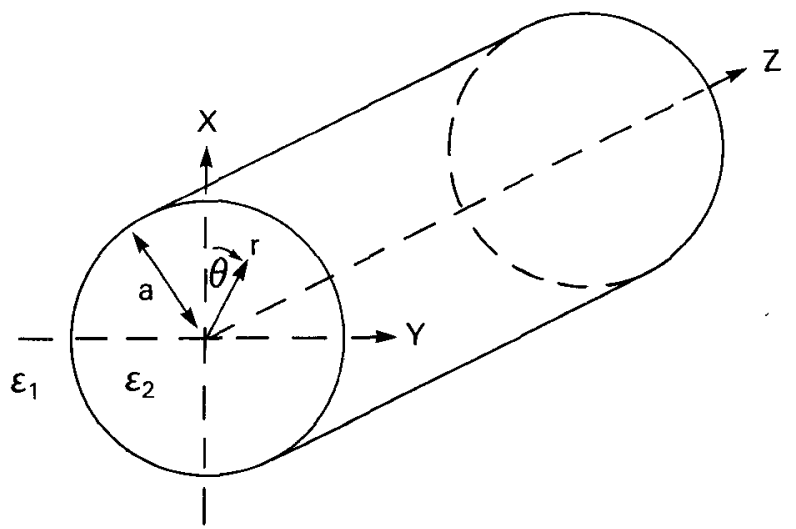

Fig. 1. Section of a dielectric waveguide.

For a circular dielectric rod in free space, the power attenuation is given by

$$
\alpha=\frac{2 \pi}{\lambda_{0}} \epsilon_{r} \tan \delta R
$$

where

$$
R=\frac{\int_{0}^{2 \pi} \int_{0}^{a}(\mathbf{E} \cdot \mathbf{E}) \rho d \rho d \theta}{\eta_{0} \int_{0}^{2 \pi} \int_{0}^{\infty}\left(E_{\rho} H_{\theta}-H_{\theta} E_{\rho}\right) \rho d \rho d \theta}
$$

where $E$ and $H$ are given by Stratton [1], and $\eta_{0}=$ $\left(\mu_{0} / \epsilon_{0}\right)^{1 / 2}$ is the impedance of free space.

$R$ has been calculated for the $\mathrm{HE}_{11}$ mode by Elsasser [3], and is sketched for two common values of $\epsilon_{r}$ in Fig. 2 . Note that the factor $R$ falls rapidly as the diameter $d$ of the rod is decreased. This reflects the decrease in attenuation as the relative amount of power within the rod falls.

In the opposite limit, as $d \rightarrow \infty, R$ approaches the value $\frac{1}{\sqrt{\epsilon_{r}}}$. This corresponds to all of the power in the $\mathrm{HE}_{11}$ mode propagating within the dielectric. In this limit, (2) and (3) are equal.

Finally, the power propagating along the dielectric rod decays as

$$
P(z)=P_{0} e^{-\alpha z} .
$$

In a differential length $\Delta z$, the power loss is given by

$$
\Delta P=\alpha P \Delta z \text {. }
$$

This power loss will not be uniform across the cross section of the guide, but will depend on the field configurations for the $\mathrm{HE}_{11}$ mode. However, in later discussions it will be assumed that this power loss is a uniform source of heat across the cross section of the guide.

\section{Dielectric BREAKDown}

Dielectric breakdown will occur whenever the electric field within the dielectric rod exceeds a certain critical value. In order to determine the maximum power that can be handled before breakdown occurs, it is necessary to relate the maximum electric field $E_{0}$ in the dielectric rod to the transmitted power $P$.

Marcuse has derived the relationship between $E_{0}$ and $P$ for the $\mathrm{HE}_{11}$ mode [4]. However, the resulting expression is

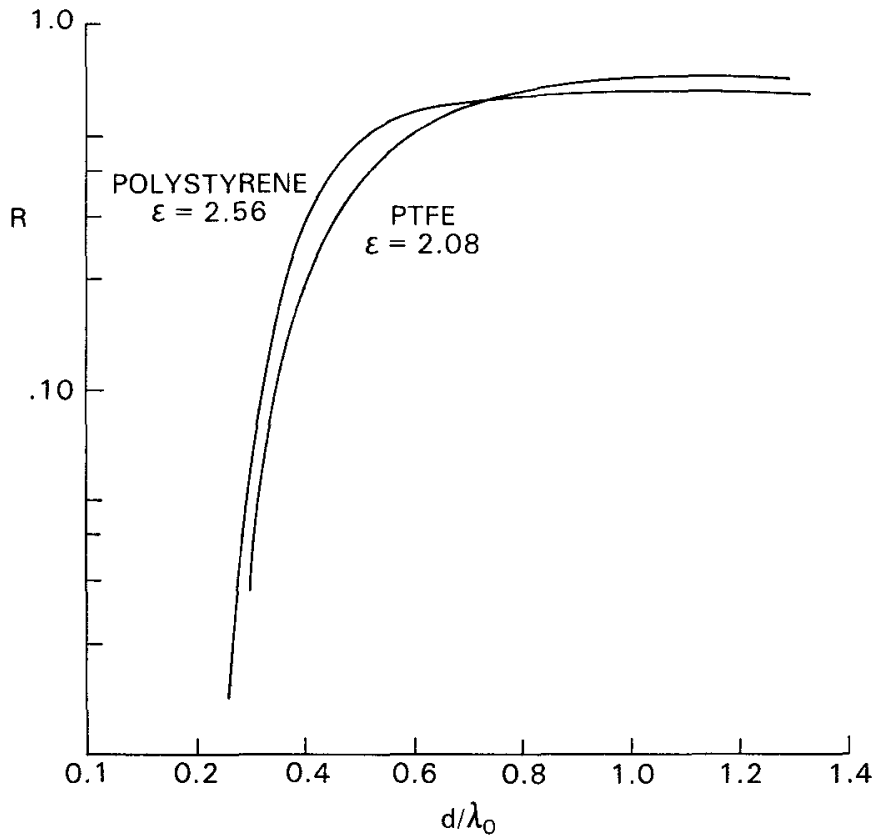

Fig. 2. $R$ as a function of waveguide diameter.

quite complicated and is inconvenient to evaluate for any specific instance. In addition to the evaluation of numerous Bessel and Hankel functions, one must solve a transcendental equation to obtain the guide wavelength of the mode for a specific set of parameters. In light of the simplifying assumptions to be made elsewhere in this discussion, the effort to evaluate $E_{0}$ versus $P$ for the $\mathrm{HE}_{11}$ mode is not warranted.

As an alternative, consider the simple calculation of the maximum electric field in the $\mathrm{TE}_{10}$ mode of a dielectricfilled rectangular metal waveguide. In practice, the $\mathrm{HE}_{11}$ mode of the dielectric rod is often launched from the $\mathrm{TE}_{10}$ rectangular mode. If the impedance match is good, the net power transferred to the dielectric rod will be comparable with the total power in the $\mathrm{TE}_{10}$ mode.

As the $\mathrm{HE}_{11}$ mode is launched, the power density $\mathbf{E} \times \mathbf{H}$ diminishes as energy spreads into the evanescent fields around the dielectric rod. Thus, the relation between $E_{0}$ and $P$ in the section of dielectric-filled rectangular waveguide should serve as an upper limit in the consideration of dielectric breakdown for a given power level. In practice, there will often be an air gap at the sides of the dielectric at the point where it is inserted into the metal guide. Since the dielectric breakdown strength of air is often less than that of a dielectric, it is also important to compute the relation between $E_{0}$ and $P$ for an air-filled waveguide.

For a dielectric-filled rectangular waveguide, the $\mathrm{TE}_{10}$ mode is given by [5]

$$
E_{y}=E_{0} \sin \frac{\pi x}{a}
$$

where $E_{0}$ is the peak electric field and $a$ is the width of the rectangular guide. The transmitted power is found from

$$
P=\frac{1}{2 Z} \int_{0}^{b} \int_{0}^{a} E_{y}^{2} d x d y
$$

where $a$ and $b$ are the waveguide dimensions, and the 
impedance $Z$ is given by

$$
Z=\frac{n}{\left[1-\left(\omega_{c} / \omega\right)^{2}\right]^{1 / 2}}
$$

$\eta=\left(\mu_{0} / \epsilon\right)^{1 / 2}$ and $\omega_{c}$ is the cutoff frequency of the waveguide.

Integrating (8), the transmitted power becomes

$$
P=\frac{1}{4} \sqrt{\epsilon_{r}} \frac{\left[1-\left(\omega_{c} / \omega\right)^{2}\right]^{1 / 2}}{\eta_{0}} a b E_{0}^{2}
$$

where $\eta_{0}=\left(\mu_{0} / \epsilon_{0}\right)^{1 / 2}=377 \Omega$, and $\epsilon_{r}=\epsilon / \epsilon_{0}$.

To launch the $\mathrm{HE}_{11}$ mode on a dielectric rod operated at $70 \mathrm{GHz}$, one would most likely use a $V$-band WR15 waveguide having inside dimensions of $3.78 \times 1.88 \mathrm{~mm}^{2}$ and a cutoff frequency of $39.863 \mathrm{GHz}[6]$. For a PTFE rod with an $\epsilon_{r}$ of 2.08 [7] and a dielectric strength of $1.7 \times 10^{7}$ $\mathrm{V} / \mathrm{m}$ [8], the power level at which breakdown will occur within the rectangular guide will be $P=1.6 \times 10^{6} \mathrm{~W}$. Results for a polystyrene rod will be similar.

For an air-filled guide with $\epsilon_{r}=1$ and a dielectric strength of $3 \times 10^{6} \mathrm{~V} / \mathrm{m}$, the corresponding power level will be $P=3.5 \times 10^{4} \mathrm{~W}$. Thus, it is possible that dielectric breakdown in the air-filled section of rectangular guide prior to the dielectric rod may prove to be the limiting factor. Furthermore, in some instances there might be dielectric breakdown in the evanescent field regions in the air surrounding a dielectric guide.

However, until high-power millimeter-wave sources become ávailable, these problems are essentially moot. Furthermore, little information is available about the dielectric strengths of materials at these frequencies, and the numbers used in the previous calculations may need to be modified.

\section{Dielectric Heating}

The problem of dielectric heating can be solved approximately to yield a rough estimate of another limiting factor to the power-handling capabilities of a dielectric rod. The approach used here will be to find the power level that will cause the temperature at the center of the dielectric rod to reach its melting point. The electromagnetic energy absorbed in the rod will be treated as a uniform source of heat throughout a cross section of the rod, as discussed in Section II. The heat diffuses to the outside of the rod, where it is dissipated by radiation and convection.

In the case of a dielectric waveguide, (6) gives the heat generated in a section of waveguide of length $\Delta z$ as

$$
q=\Delta P=\alpha P \Delta z
$$

where $\alpha$ is the power attenuation per unit length and $P$ is the amount of power propagating down the rod.

To predict the limits imposed by dielectric heating, it is necessary to find $P$ as a function of $T_{0}$, the temperature at the center of the rod. The maximum value of $P$ will be determined by setting $T_{0}=T_{m}$, the melting temperature of the dielectric. (Depending on the choice of dielectric, $T_{m}$ may actually correspond to the glass transition temperature of a polymer, as opposed to a "melting" temperature.)
Two important assumptions will be made. The first is that heating across the cross section of the dielectric is uniform. Although this is not strictly accurate, it results in a significant simplification of the problem, and provides a useful estimate of the maximum power level that can be tolerated.

The second is that the temperature is approximately constant over the cross section of the rod. That is, the temperature $T_{0}$ at the center of the rod is approximately equal to the temperature $T_{w}$ at the wall of the guide. This will typically be true for a small diameter dielectric rod, where convection and radiation processes at the surface of the guide are much less efficient than thermal conduction within the rod.

It is now necessary to relate the temperature $T_{w}$ at the wall of the dielectric rod to the ambient temperature $T_{\infty}$. This is done with the help of equivalent heat transfer coefficients.

Convection can be characterized by a heat transfer coefficient $h_{c}$ such that

$$
q_{c}=h_{c} A\left(T_{W}-T_{\infty}\right)
$$

where $q_{c}$ is the heat lost to convection, and $A$ is the area of the convecting surface.

Thermal radiation is characterized by the Stefan-Boltzman radiation law, where

$$
q_{r}=\xi \sigma A\left(T_{w}^{4}-T_{\infty}^{4}\right) .
$$

The constant $\sigma$ is equal to $5.67 \times 10^{-8} \mathrm{~W} / \mathrm{m}^{2} \mathrm{~K}^{4}$, and $\xi$ is the emissivity of the radiating surface.

Equation (13) can be rewritten as

$$
q_{r}=h_{r} A\left(T_{w}-T_{\infty}\right)
$$

where

$$
h_{r}=\xi\left(T_{w}+T_{\infty}\right)\left(T_{w}^{2}+T_{\infty}^{2}\right) .
$$

The total heat $q$ in a rod element of volume $\pi \mathrm{a}^{2} \Delta z$ is given by (11). Combining (11)-(13)

$$
\alpha P \Delta z=h_{t}(2 \pi a \Delta z)\left(T_{w}-T_{\infty}\right)
$$

where

$$
h_{t}=h_{r}+h_{c}
$$

Thus

$$
T_{w}=\frac{\alpha P}{2 \pi a h_{t}}+T_{\infty}
$$

This expresses the temperature at the wall of the rod in terms of the power carried by the $\mathrm{HE}_{11}$ mode, the loss factor $\alpha$, and the combined effects of thermal radiation and convection at the surface of the rod.

The heat transfer coefficient $h_{r}$ is given by (15). Holman [9] gives an expression for $h_{c}$ due to free convection across a horizontal cylinder, where

$$
h_{c}=1.32\left\{\left(T_{w}-T_{\infty}\right) / 2 a\right\}^{1 / 4} \frac{W}{m^{2} K}
$$

and $\left(T_{w}-T_{\infty}\right)$ is specified in degrees centigrade. (Other 
TABLE I

Properties of Polystyrene and PTFE

\begin{tabular}{|c|c|c|}
\hline & POLYSTYRENE & PTFE \\
\hline$\varepsilon_{r}(7)^{*}$ & 253 & 200 \\
\hline TAN $d(7)^{*}$ & 00100 & 00021 \\
\hline $\mathrm{d}_{\text {CRITICAL }}-\mathrm{mm}$ & 255 & 316 \\
\hline TMeLting $-{ }^{\circ} \mathrm{C}(10)$ & 240 & 327 \\
\hline$T_{\text {GLASS }}={ }^{\circ} \mathrm{C}(10)$ & 100 & - \\
\hline$k-W / m K^{(8)} \cdots$ & 116 & 277 \\
\hline $\begin{array}{l}\text { DIELECTRIC } \\
\text { STRENGTH }-v \text { volt } / \mathrm{m}^{(8)}\end{array}$ & $17 \times 10^{7}$ & $197 \times 10^{7}$ \\
\hline $\begin{array}{l}- \text { measured at approx } 7 \\
+ \text { measured at } 50{ }^{\circ} \mathrm{C}\end{array}$ & $\mathrm{Hz}{ }^{(7)}$ & \\
\hline
\end{tabular}

TABLE II

Power-Handling Capacities of Various Dielectric WAVEGUIDES AT $71.7 \mathrm{GHz}[7]$

\begin{tabular}{|c|c|c|c|c|c|c|c|c|c|}
\hline MATERIAL & $\begin{array}{l}\text { DIAMETER } \\
(\mathrm{mm})\end{array}$ & $\mathrm{R}$ & $\begin{array}{c}\alpha \\
\text { NEPER } / m\end{array}$ & $\begin{array}{c}\mathrm{h}_{\mathrm{C}} \\
\text { WATT } / \mathrm{m}^{2}\end{array}$ & $\begin{array}{c}h_{R} / \xi \\
K \text { WATT } / m^{2} k\end{array}$ & $\begin{array}{c}T_{\infty} \\
\text { KELVIN }\end{array}$ & $\begin{array}{l}\text { Prad } \\
\text { WATTS }\end{array}$ & $\begin{array}{l}\text { Pconv } \\
\text { WATTS }\end{array}$ & $h_{t} a / 2 k$ \\
\hline PTFE & 201 & 3253 & 2080 & 2948 & 1469 & 100 & 2171 & - & 080 \\
\hline PTFE & 201 & 3253 & 2080 & 2610 & 2257 & 293 & 2021 & 2322 & 088 \\
\hline PTFE & 3.00 & 6001 & 3838 & 2667 & 14.69 & 100 & 1734 & - & 112 \\
\hline PTFE & 300 & 6001 & 3838 & 2361 & 2257 & 293 & 1604 & 1673 & 125 \\
\hline POLYSTYRENE & 160 & 2251 & 8671 & 2683 & 400 & 100 & 62 & - & 106 \\
\hline POLYSTYRENE & 160 & 2251 & 8671 & 1974 & 850 & 293 & 38 & 86 & 097 \\
\hline POLYSTYRENE & 238 & 5438 & 20948 & 2429 & 400 & 100 & 38 & - & 145 \\
\hline POLYSTYRENE & 238 & 5438 & 20948 & 1787 & 850 & 293 & 23 & 47 & 135 \\
\hline
\end{tabular}

expressions are available for $h_{c}$ under conditions of forced convection, convection from a vertical cylinder, etc., [9].)

A final problem concerns the measurement of the emissivity of a dielectric. However, since $0 \leqslant \xi \leqslant 1$, it is possible to determine the limits of thermal radiation even if accurate data for $\xi$ is not available.

It is now possible to estimate the power-handling capabilities of practical millimeter-wavelength dielectric-rod waveguides. Table I gives relevant properties of PTFE and polystyrene from (7), (8), and (10). There is some slight disagreement in the literature over some of these values. This is not surprising, as polymers are noted for sampleto-sample variations of their properties. Furthermore, some parameters, such as $\xi, \tan \delta$, and $\mathrm{k}$, the thermal conductivity of the dielectric, exhibit variations with temperature. However, the values presented in Table I are more than adequate for the problem at hand.

Table II presents calculations of power dissipation under a variety of conditions for PTFE and polystyrene waveguides of various diameters. The assumption is made that $T_{W}=T_{0}=T_{m}$, the melting temperature of PTFE, and $T_{w}=$ $T_{0}=T_{g}$, the glass transition temperature of polystyrene. A figure of merit, $h_{t} a / 2 k$, is also presented. Solution of the classic problem of heat flow in a cylinder indicates that if this quantity is substantially less than 1 , the assumption that $T_{0} \simeq T_{w}$ is reasonable [9].

The power required to set $T_{0}$ equal to $T_{m}$ or $T_{g}$ is calculated separately for convection only $\left(h_{r}=0\right)$ and for radiation only $\left(\xi=1, h_{c}=0\right)$. Two values of ambient temperature are considered. $T=293 \mathrm{~K}$ corresponds to room temperature, $T=100 \mathrm{~K}$ to a hypothetical space-born environment. In this latter case, heat loss due to convection is assumed to be zero.

The important conclusion to be drawn from Table II is that the maximum power levels permitted by the consideration of dielectric heating are substantially less than those calculated for dielectric breakdown. Furthermore, there is a huge difference between the power that can be handled by a PTFE rod and that for a polystyrene rod. This difference results from the combined effects of the different values for $\tan \delta$ and $T_{g}$ or $T_{m}$. With the continued development of high-power millimeter-wave sources, it is conceivable that polystyrene will be unsatisfactory for many applications. PTFE, on the other hand, has much to recommend it. It is already commerically available as clad cylindrical dielectric waveguide for use in frequency bands from $26-140 \mathrm{GHz}$ [11].

Finally, it should be noted that the effective surface area of a dielectric waveguide can be increased by roughening the surface. This will increase the amount of thermal radiation and the efficiency of convection. An imperfect surface is often thought to result in unwanted microwave radiation from the dielectric rod. However, Marcuse has shown that a rough surface causes radiation loss only when the spatial dimensions of the surface irregularities are comparable with the difference in wavelengths between 
guided and radiation modes of the dielectric rod [12]. Thus, it may well be practical to modify the surface finish of a dielectric rod for heat dissipation purposes without interfering with its microwave performance.

\section{Conclusion}

The previous discussion has been an attempt to compare the effects of dielectric breakdown and dielectric heating in a dielectric waveguide. Sample calculations have been presented for polystyrene and PTFE guides operated in the $70-\mathrm{GHz}$ region. The results indicate that dielectric heating, not breakdown, limits the amount of power that a typical rod waveguide can carry.

The PTFE rod can carry considerably more power than its polystyrene counterpart. Coupled with desirable mechanical properties, such as strength, flexibility, and corrosion resistance, the dielectric and thermal properties of PTFE suggest that it will be an important material in the development of high-power millimeter-wave technology.

Traditionally, the heat transfer calculations presented in Section IV have been of interest primarily to mechanical engineers. However, given the close relationship between classical heat flow and electrostatics, the discipline of heat transfer should perhaps be of more interest to electrical engineers. At least in the case of millimeter-wavelength dielectric waveguides, the heat transfer calculations appear to add some useful insight to the practical problems that will eventually be encountered as millimeter-wave technology progresses.

\section{ACKNOWLEDGMENT}

The author would like to recognize A. D. Krall at the Naval Surface Weapons Center, Professor F. R. Morgenthaler of M.I.T., and K. D. Hall, Jr., for their considerable assistance with the study of dielectric waveguide and concepts of heat transfer.

\section{REFERENCES}

[1] J. R. Stratton, Electromagnetic Theory. New York: McGraw-Hill, 1941.

[2] A. L. Cullen and E. F. F. Gillespie, "A new method for dielectric measurements at millimeter wavelengths," presented at Symp. on Millimeter Waves, Polytechnic Institute of Brooklyn, Apr. 1959.

[3] W. M. Elsasser, "Attenuation in a dielectric circular rod," J. App. Phys., vol. 20, p. 1193, Dec. 1949.

[4] D. Marcuse, "Radiation losses of the dominant mode in round dielectric waveguides,"' Bell Syst. Tech. J., pp. 1665-1693, Oct. 1970.

[5] E. C. Jordan and K. G. Balmain, Electromagnetic Waves and Radiating Systems. Englewood Cliffs, NJ: Prentice-Hall, 1968.

[6] TRG Millimeter Waves Products Catalog, 1976.

[7] D. G. Jablonski, "Attenuation of circular dielectric waveguide at millimeter wavelengths," IEEE Trans. Microwave Theory Tech., vol. MTT-26, pp. 667-671, Sept. 1978.

[8] J. Agranoff, Ed., Modern Plastics Encyclopedia. New York: McGraw-Hill, 1976.

[9] J. P. Holman, Heat Transfer, 4th ed. New York: McGraw-Hill, 1976.

[10] J. Brandrup and E. H. Immergut, Eds., Polymer Handbook. New York: Wiley, 1975.

[11] Gore-Tex Dielectric Waveguide Assemblies. (Gore-Tex is a registered trademark of W. L. Gore \& Associates, Newark, DE)

[12] D. Marcuse, "Radiation losses of dielectric waveguides in terms of the power spectrum of the wall distortion function," Bell Syst. Tech. $J$, vol, 48, no. 10, pp. 3233-3242, Dec. 1969.

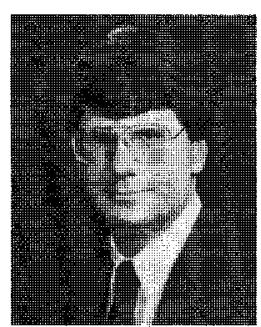

Daniel Jablonski (M'83-SM'84) was born in Washington, DC, in 1954. He received the B.S. and M.S. degrees in electrical engineering from M.I.T., Cambridge, MA, in 1976 and 1977, respectively, and the Ph.D. degree in physics from Cambridge University, England, in 1982.

As a research physicist at the Naval Surface Weapons Center in White Oak, MD, his technical interests include superconducting electronics, millimeter waves, and the design of special-purpose sensor systems. 